\title{
EchoGéo
}

$13 \mid 2010$

Afrique, 50 ans d'indépendance : État et territoires

\section{Pourquoi croire au modèle du développement territorial au Maghreb ? Une approche critique}

Kirsten Koop, Pierre-Antoine Landel et Bernard Pecqueur

\section{OpenEdition}

\section{Journals}

Édition électronique

URL : https://journals.openedition.org/echogeo/12065

DOI : 10.4000/echogeo.12065

ISSN : 1963-1197

Éditeur

Pôle de recherche pour l'organisation et la diffusion de l'information géographique (CNRS UMR 8586)

Référence électronique

Kirsten Koop, Pierre-Antoine Landel et Bernard Pecqueur, « Pourquoi croire au modèle du développement territorial au Maghreb ? Une approche critique », EchoGéo [En ligne], 13 | 2010, mis en ligne le 20 septembre 2010, consulté le 03 août 2021. URL : http://journals.openedition.org/echogeo/ 12065 ; DOI : https://doi.org/10.4000/echogeo.12065

\section{Ce document a été généré automatiquement le 3 août 2021}

EchoGéo est mis à disposition selon les termes de la licence Creative Commons Attribution - Pas d'Utilisation Commerciale - Pas de Modification 4.0 International (CC BY-NC-ND) 


\title{
Pourquoi croire au modèle du développement territorial au Maghreb ? Une approche critique
}

\author{
Kirsten Koop, Pierre-Antoine Landel et Bernard Pecqueur
}

\section{Introduction}

Depuis la décolonisation ${ }^{1}$, les politiques de développement économique des pays du Maghreb, ont été fortement déterminées par des modèles extérieurs (occidentaux ou socialistes), axés principalement sur la croissance et supposés être mis en œuvre par les Etats avec l'appui des dispositifs de coopération. Après une phase où étaient préconisées planification centralisée et industrialisation (Maroc, Algérie, Tunisie), vint celle $\mathrm{du}$ « développement local ». Nous assistons actuellement au transfert du modèle français de « développement territorial » vers ses anciennes colonies.

Dans le contexte contemporain des processus de la mondialisation, les sciences régionales des pays industrialisés (économie régionale et géographie économique notamment) semblent avoir découvert le «territoire $»^{2}$ à l'échelle infranationale, comme «nœud des processus critiques de développement et de croissance " (Scott, 2003). Le territoire est désormais considéré comme le gabarit adéquat à la conception du développement et devient ainsi le cadre de l'action publique. Cela implique la mutation du rôle de l'Etat, moins régulateur, et l'amplification de la décentralisation. En France, le concept de " projet de territoire » est devenu référent incontournable de l'action publique. Depuis le milieu des années 2000, la DATAR ${ }^{3}$ a développé des actions de coopération avec les pays du Maghreb, d'abord au Maroc, puis en Tunisie, et enfin en Algérie, pour accompagner une profonde mutation des politiques publiques, reposant sur l'affirmation du «territoire de projet» comme espace et mode pertinent du développement. Au vu des difficultés, voire de l'échec des transferts antécédents de modèles de développement occidentaux, il semble de bon sens de questionner les conditions et limites de la «transférabilité » de ce nouveau modèle vers des pays du 
Sud. Si les programmes de coopération mis en œuvre par les Etats attestent cette possibilité de transfert, la réalité concrète de leur mise en œuvre, dans des contextes qui diffèrent considérablement des cadres de référence français, souligne la difficulté de l'exercice.

\section{L'évolution des visions françaises de développement et leurs applications au Maghreb}

\section{Le rattrapage industriel}

Les programmes et plans de développement conçus par et pour les pays maghrébins depuis leur indépendance n'ont pas échappé à l'inscription, directe ou indirecte, dans la vision et le modèle français de développement. Le référentiel dominant des politiques nationales, aussi variables furent-elles ${ }^{4}$, était celui du rattrapage du retard par rapport aux pays industrialisés. Les gouvernements des différents pays du Maghreb fondaient leurs politiques de développement sur l'industrialisation et la planification centralisée et nationale (Dresch, 1963). Tous les éléments de base de la vision moderniste du développement peuvent être retrouvés : le « développement » fut pensé comme une rupture avec le "sous-développement", il était supposé être réalisé à travers la croissance du secteur économique moderne, les particularités politiques, les réalités socioculturelles diverses et le potentiel du secteur informel grandissant étant négligés par principe.

Certes, ce fond commun dans les conceptions du développement se décline de façons différentes dans ces pays. Les programmes nationaux successifs des années 1960 et 1970 montrent une forte préoccupation pour le renforcement des pôles de croissance ${ }^{5}$, dans une approche sectorielle, le gabarit d'application étant la nation entière (Scott, Storper, 1991, p. 8). De cette approche du développement découlaient aussi les modalités du découpage régional : celui-ci fut réalisé sur la base d'un ensemble de critères largement dominés par les aspects économiques et dans une démarche administrative, servant surtout à permettre l'application des politiques nationales (voir, par exemple, Bellaoui, 2009).

\section{Le développement local}

Le bilan de l'application de ces stratégies modernistes au Maghreb audébut des années 1980 est connu: des chiffres de croissance économique parfois négatifs (Algérie), un fort endettement, la persistance de la pauvreté de masse, surtout en milieu rural. Les pôles de croissance manquaient de dynamisme, les effets d'entraînement se laissaient attendre. Les causes de l'échec seront tantôt cherchés dans les facteurs internes aux pays (régimes de dictature, corruption, clientélisme), tantôt dans les facteurs externes, comme l'échange inégal entre pays industrialisés et pays en développement. Des économistes, s'inscrivant dans les théories de la dépendance, découvrent les surcoûts de l'industrialisation liés à la dépendance des transferts financiers et du savoir-faire occidental (Courlet, Gaulet, 1973). Ils critiquent l'image statique que les analyses modernistes aussi bien que marxistes ont des sociétés « traditionnelles », leur vision ahistorique et découvrent la petite production artisanale et le secteur informel non 
comme "arriéré » et "obstacle " au développement, mais comme son potentiel (Courlet, Judet, 1986, p. 522).

Ainsi émerge au sein des sciences économiques un courant scientifique se penchant sur le potentiel économique des régions maghrébines (Bouchara, 1987 ; Courlet 1989, etc.). Il est intéressant de noter que les auteurs de ce nouveau type d'études régionales analysent, en parallèle, la reconversion réussie de certaines régions industrielles occidentales en crise, notamment de l'Italie du Nord (Judet, 1989; Garofoli, 1986) ainsi que le succès des petits tigres asiatiques (Judet, 1986). Dans ce va-et-vient entre études au Nord et aux Suds commence à se dessiner une nouvelle vision du développement : celui du "développement endogène». Rapidement qualifié de développement local (Pecqueur, 1989 ; Colettis et al. 1990), il donne toute sa place aux dynamiques locales, aux acteurs locaux, à leur initiative et aux stratégies endogènes ayant échappé à la planification dite "d'en haut», a-spatiale dans son approche. Cette découverte des dynamiques locales s'inscrit, certes, dans un courant plus vaste, international, travaillant aussi bien sur les pays du Nord dans le contexte post-fordiste qui se dessine, que sur les pays des Suds. Elle trouve des fondements théoriques dans les travaux internationalement reconnus de Stöhr et Tödling (1971), Friedmann et Weaver (1979) et Stöhr et Taylor (1981).Au Maghreb, elle sera particulièrement diffusée à partir de la conférence fondatrice sur le "développement endogène », organisée par l'Institut de Recherche Economique et de Planification (IREP) à Tunis en 1987.

Ce changement de perspective scientifique trouve son application concrète aussi bien en France que dans les pays du Maghreb. Pourtant, ses formes et dimensions varient fortement. En France, en réaction à la crise du modèle fordiste, émerge, à la fin des années 1980 , le "projet de territoire ", outil qui permet à des acteurs coordonnés d'apporter des solutions à des problèmes productifs inédits à l'échelle infra-nationale (Pecqueur, 1989, p. 15). Cette approche définit le territoire "comme le résultat d'un processus de construction par les acteurs » (Pecqueur, 2005, p. 299). Les relations sont placées au cœur du système d'innovation et développées à des échelles intermédiaires qui se situent entre l'individu et les systèmes productifs nationaux. Le cadre de l'Etat nation, comme espace de développement et de mise en œuvre des politiques macroéconomiques, est ainsi remplacé par un espace méso-économique. Celui-ci est appréhendé selon deux modes : celui de la coordination sectorielle, entre acteurs d'une même filière (Gillard, 1970) et celui du regroupement partiel d'acteurs ou d'agents économiques, dans des logiques de coopération (Gilly et al., 2004). En réaction aux dépendances financières et organisationnelles, l'autonomie est au cœur des notions de "self-reliance " ou de développement autocentré, qui repose sur "l'utilisation prioritaire des facteurs de production disponibles localement » (Rist, 2007, p. 219).

Contrairement à l'application en France dece changement de vision sur les conditions du développement dans une partie du monde académique, il n'influençait guère les politiques nationales maghrébines. Des projets participatifs de « développement d'en bas " émergent cependant timidement dans le milieu de la coopération et des associations à partir de la fin des années 1980. Alors que les processus de décentralisation, base indispensable pour la réalisation du "développement local», s'approfondissent en France, ils sont encore loin de s'affirmer au Maghreb. 


\section{Le développement territorial}

A partir du milieu des années 1990, le concept du développement local commence donc à être remplacé, en France, par celui du développement territorial, concept transféré au Maghreb depuis les années 2000 par le biais de programmes bilatéraux de coopération. En quoi consiste le changement d'optique? Pourquoi assiste-t-on, contrairement au faible succès du modèle de développement local, à une prise en compte réelle par les autorités publiques de ce nouveau modèle?

France ou Maghreb, Nord ou Sud, pour la première fois depuis l'époque de la colonisation, les pays semblent se trouver face à des dynamiques similaires : celles de la mondialisation. Les défis semblent être les mêmes : adapter les économies et sociétés face à des marchés libéralisés et à la mobilité globale des firmes. Aussi, les réponses au changement fondamental des conditions du développement, au Nord comme au Sud, paraissent-elles être les mêmes : ouverture économique volontaire ou forcée (au Sud, à travers les programmes d'ajustement structurel), retrait de l'Etat par rapport aux logiques du marché et découverte unanime du territoire, à l'échelle infranationale, comme «l'espace d'action publique, de mobilisation politique et de changement économique " (Keating, Loughlin, 1997, p. 11). Un changement fondamental dans la conception du développement, généralisé cette fois-ci et non restreint à une certaine communauté scientifique, comme ce fut le cas pour le développement local, s'est réalisé : celui du rattrapage du retard des nations à « l'intégration des territoires » dans les flux et réseaux mondiaux (Koop, 2007). Bien que la notion de développement local continue à être utilisée, certains auteurs et acteurs du développement passent à celle du développement territorial pour des raisons non explicitées, mais consistantes.

Le premier argument à avancer est que le développement local est trop connoté, autour d'un développement économique autocentré. A l'ère de la mondialisation et du libre échange, le développement local doit désormais être pensé en articulation avec d'autres échelles. Cette ouverture aux marchés introduit la nécessité pour les territoires d'être compétitifs en ajustant production et vente sur les marchés extérieurs.

La seconde différence réside dans l'émergence de nouveaux modes de gouvernance. Dans le contexte de la décentralisation et de l'affirmation de la société civile en tant qu'acteur du développement, le territoire n'est plus le monopole de l'Etat ou des acteurs politiques. D'autres acteurs le revendiquent pour régler leurs problèmes, qu'ils soient d'origineéconomique, sociale ou environnementale. Le développement territorial s'appuie sur une double notion de territoire : le territoire «donné », issu de l'histoire administrative des pays, en opposition au territoire "construit " (Colletis, Pecqueur, 1993). En parallèle à l'émergence de politiques de contractualisation visant à accompagner les processus de développement à l'échelle infranationale, la gouvernance désigne « une production de règles par des acteurs en vue de réguler le système économique, productif, social, etc.». (Angeon, Houédété, 2006). Elle place la coordination au cœur des processus de développement, les acteurs ayant une plus ou moins grande capacité à se grouper pour créer leurs propres organisations, règles et normes, face à des problèmes à résoudre localement.

La troisième différence tient au fait que les acteurs ne visent plus l'allocation optimale de ressources dont seraient dotés initialement les territoires, mais la création de ressources territoriales. Ce processus de création se fait en valorisant les potentiels 
spécifiques des territoires, c'est-à-dire les avantages relatifs qui distinguent le territoire par rapport à ses concurrents potentiels (ce processus de spécification s'illustre par exemple dans le cas des labellisations de produits agro-alimentaires en appellation d'origine). Ceux-ci vont alors "promouvoir une stratégie haute de développement reposant sur des ressources spécifiques ou latentes produites par des acteurs locaux et dédiées " (Adelmalki, Courlet, 1996, p. 18). C'est à partir de ces ressources spécifiques que les acteurs du territoire vont rechercher une compétitivité par la différenciation, à partir de qualités spécifiques, en parallèle à une mobilisation systématique des patrimoines et identités locales (Landel, 2007).

Ainsi, le développement territorial se différencie du développement local au travers de trois traits essentiels: l'ouverture des marchés, la construction de systèmes de gouvernance élargis à d'autres acteurs et la mobilisation de ressources spécifiques.

Tableau 1 - Du Développement local au développement territorial

\begin{tabular}{|l|l|}
\hline Développement local & Développement territorial \\
\hline $\begin{array}{l}\text { Renforcement de circuits locaux } \\
\text { Identités et échanges mono scalaires }\end{array}$ & $\begin{array}{l}\text { Intégration dans marchés à diverses échelles } \\
\text { Identité et échanges multi scalaires }\end{array}$ \\
\hline $\begin{array}{l}\text { Mobilisation de ressources locales en } \\
\text { réponse aux besoins locaux }\end{array}$ & $\begin{array}{l}\text { Compétitivité (locale, nationale, mondiale) par la } \\
\text { génération de ressources spécifiques }\end{array}$ \\
\hline $\begin{array}{l}\text { Rôle déterminant de l'Etat dans le dispositif } \\
\text { de contractualisation }\end{array}$ & \begin{tabular}{l} 
Intégration de nouveaux acteurs dans la gouvernance \\
\hline
\end{tabular}
\end{tabular}

\section{Les modalités de transfert contemporain du modèle du développement territorial dans les politiques publiques}

\section{Pourquoi le transfert du modèle de développement territorial français?}

Les lois françaises de 1995 (loi Pasqua) et 1999 (loi Voynet) marquent la transformation fondamentale de la politique nationale d'Aménagement du Territoire et traduisent le changement de vision de développement. On passe à une politique décentralisée, au sein de laquelle le projet de territoire intègre le diagnostic de la situation de départ, la construction d'une vision prospective et la définition d'une stratégie au travers de la contractualisation (Gorgeu, Jenkins, 1997, p. 34).

Contrairement au concept du développement local, le développement territorial en tant que modèle fait son entrée dans la politique d'aménagement des pays maghrébins. Durant la dernière décennie, trois opérations de transferts ont été décidées puis mises en œuvre au niveau des Etats. De 2001 et 2007, le Royaume du Maroc a mis en place un programme intitulé "Appui institutionnel à la mise en œuvre d'une politique d'aménagement du territoire rénovée au Maroc» avec la participation de l'Etat 
français, dans le cadre d'un projet du Fonds de Solidarité Prioritaire. La coopération avec la Tunisie s'est inscrite de 2006 à 2009 dans le cadre d'un jumelage, impulsé par la Commission européenne, et associant des partenaires français et italiens. L'objectif affiché est de «renforcer des capacités institutionnelles des organismes de développement régional en matière de promotion de l'investissement privé ». Enfin, l'Algérie bénéficie depuis 2008 d'un nouveau projet du FSP intitulé «Appui institutionnel à la mise en œuvre de la politique d'aménagement et de développement durable en Algérie ».Il s'agit d'opérations longues, mobilisant des moyens importants, impliquant au niveau français la DATAR.

Après l'échec relatif du transfert du modèle de rattrapage industriel, conçu à l'échelle nationale, qu'est-ce qui peut justifier cette nouvelle ingérence occidentale dans ses excolonies? Tout comme celui du développement local, il paraît évident que cette approche dispose d'un atout majeur par rapport aux stratégies sectorielles et nationales: elle permet la prise en compte des acteurs locaux et des logiques socioculturelles et endogènes (souvent dites « informelles »), si longtemps négligés par les politiques publiques. Il s'agit donc de profiter d'opportunités nouvelles, en élargissant le champ des possibles en matière de ressources. Même s'il s'agit d'un modèle universel d'adaptation à la mondialisation, l'application de la stratégie du développement territorial paraît particulièrement séduisante pour les Suds, car elle ouvre une voie pour échapper à la lourdecontrainte de la compétitivité globale, en mettant en avant des ressources spécifiques localisées. Ce modèle paraît donc potentiellement bien adapté aux régions en marge.

\section{Les limites du transfert}

Cette approche du développement met en évidence la nécessité d'une organisation administrative permettant l'émergence de ces projets de territoire à différentes échelles. Elle implique notamment l'autonomie administrative ainsi que l'intégration d'acteurs locaux non publics dans les processus de décision et de réalisation de projets de territoire. Un rapprochement avec la réalité des tendances d'évolution des dispositifs nationaux s'avère ainsi intéressant. Depuis 2006, une approche comparative des processus de territorialisation est engagée dans les pays méditerranéens ${ }^{6}$. Une première synthèse des travaux permet de souligner différentes dynamiques, susceptibles d'être mises en rapport avec les transferts organisés dans le champ du développement territorial.

\section{Un processus de décentralisation inachevé}

La comparaison des processus de déconcentration/décentralisation dans les trois pays du Maghreb montre que le processus de déconcentration y est largement avancé. Il se déploie jusqu'au niveau des communes, voire au niveau infra communal, avec au Maroc, la représentation du Ministère de l'Intérieur au niveau des quartiers et des douars au travers $\mathrm{du}$ "Moqaddem ». Mais la décentralisation reste incomplète. Les exécutifs des assemblées restent largement dominés par l'omniprésence du représentant de l'Etat, qui exerce un contrôle a priori sur l'ensemble des décisions, et ce à tous les niveaux. La régionalisation, comme transfert des compétences d'aménagement et de planification à un échelon intermédiaire entre l'Etat et les municipalités et instauration de l'autonomie des services d'administration aux échelles 
sous-nationales, reste embryonnaire. Cela est particulièrement le cas en Algérie et en Tunisie, où elle reste un niveau d'étude et éventuellement de planification, mais en aucun cas de décision. L'autonomie financière des collectivités locales reste très limitée. L'organisation administrative s'inscrit dans des systèmes très fortement hiérarchisés qui limitent les possibilités d'initiatives et de coordinations propres aux territoires. Enfin, la culture administrative centralisée limite la possibilité pour les acteurs de définir leurs propres principes et règles d'organisation.

De surcroît, les modalités de transfert de compétences dans les trois programmes de coopération cités, ne semblaient pas en mesure de compenser ce manque d'autonomie : les échelles décentralisées ne furent pas impliquées, à l'exception de l'expérimentation d'un diagnostic stratégique de territoire développée en 2009 sur le gouvernorat de Tozeur, pilotée par un comité composé d'administrations de l'Etat, d'élus, de chefs d'entreprises et d'associations?

\section{L'intégration des acteurs non publics}

Concernant la démarche d'intégration d'acteurs non étatiques dans le développement territorial, permettant potentiellement de construire des ressources spécifiques intégrant la réalité socioculturelle et l'histoire locale, deux constats peuvent être faits.

Au Maghreb, les associations et les ONG se sont partiellement substituées à l'Etat là où il faisait défaut, à partir des années 1980. Différents indicateurs permettent de représenter cette évolution. A titre d'exemple, l'Algérie a vu le nombre d'associations passer de 11000 à 57000 entre 1987 et 1997, puis à 73000 en 2004. Au Maroc, après une longue période où elles étaient suspectées, et face aux exigences des bailleurs de fonds internationaux, les associations ont pu se développer quelque peu. Une étude comparative réalisée au niveau local montre l'émergence d'associations de vallées, de corporations, à côté des structures traditionnelles intervenant au niveau des douars. Les partenariats se nouent avec des structures nationales et internationales. En Tunisie, à côté de la reconnaissance de la société civile au travers des conseils locaux de développement, différents types d'organisation, telles que les coopératives de service agricole, les groupements d'intérêt collectif pour la gestion des ressources naturelles ou des groupements de développement voient le jour à différentes échelles territoriales. Affichant leur orientation vers un développement participatif, les ONG suscitent elles aussi la création d'associations au niveau local, en intégrant de façon croissante des exigences de durabilité. Il s'agit là d'acteurs potentiellement déterminants des projets de territoire. L'intégration de ces dynamiques de la société civile par les autorités publiques est encore très loin d'être systématique. Aussi, les programmes de transfert de compétences éludent la question: les associations n'ont été que rarement impliquées dans la formation, et les outils et méthodes d'animation permettant leur implication font largement défaut.

Contrairement aux administrations des pays industrialisés, celles des pays des Suds, et notamment du Maghreb, se retrouvent face à un défi supplémentaire si elles veulent créer des conditions favorables à la réalisation de projets de territoire : elles doivent intégrer les notables et institutions coutumières en tant qu'acteurs stratégiques à part égale dans les politiques territorialisées. Un premier pas dans cette direction est certes la reconnaissance du douar, défini comme un « ensemble de foyers réunis par les liens réels ou fictifs de la parenté, correspondant à une cellule territoriale, comportant ou non des modes d'exploitation communautaire» (Mahdi, 2009), comme un espace 
d'intervention. Il y a là un lieu de rencontre entre les structures administratives d'une part, et les organisations tribales ou coutumières. C'est à ce niveau que l'Etat algérien a décidé de concentrer ses moyens d'intervention en faveur du développement rural au travers des Plans de Proximité et de développement Rural (P.P.D.R.) mis en œuvre depuis 2004, pour rapprocher l'Etat des populations qui en sont éloignées.

$\mathrm{Au}$ final, cette faible «autonomie» territoriale peut être illustrée par le schéma suivant :

Schéma 1 - Autonomie du secteur public aux échelles infra nationales et intégration des acteurs non publics : deux processus en cours mais inachevés

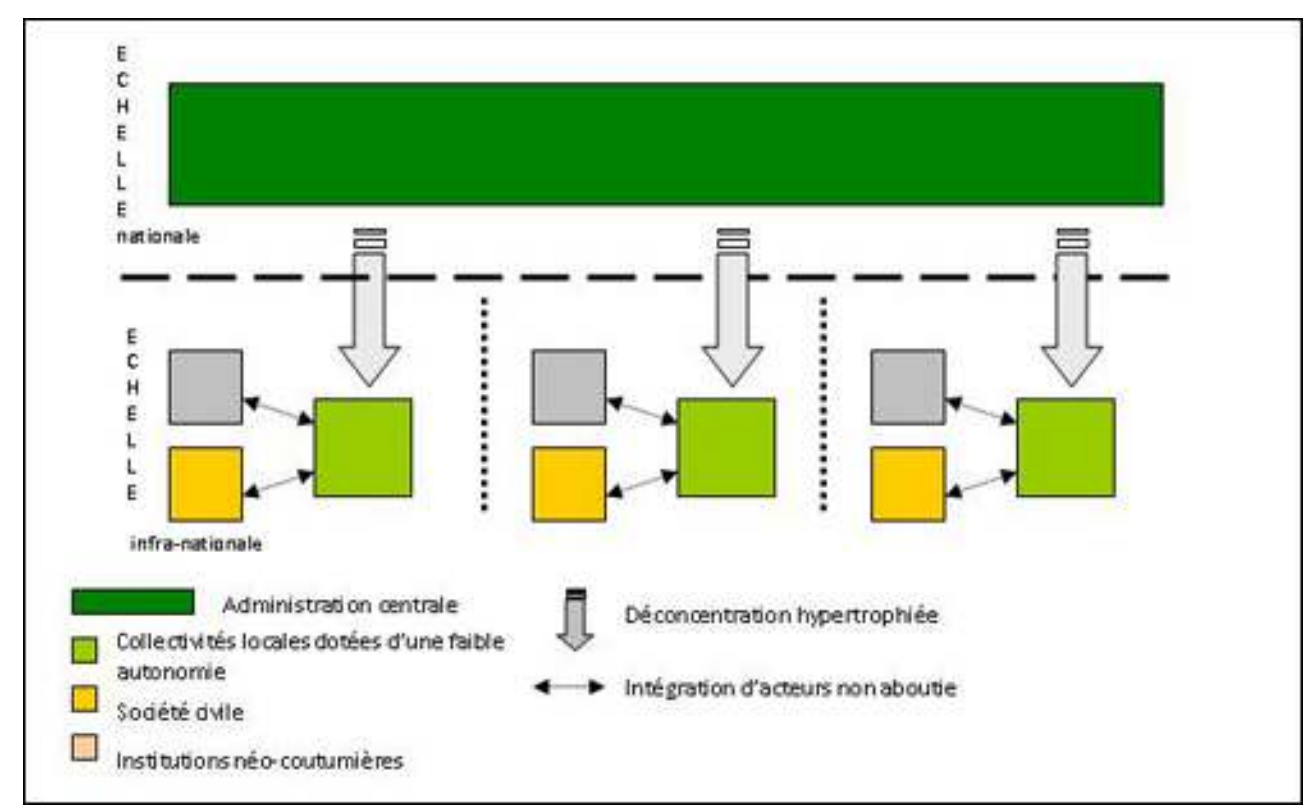

Auteurs : Kisten Koop, Pierre-Antoine Landel, Bernard Pecqueur

Parallèlement à ces processus administratifs, bien qu'incomplets, ouvrant la voie à la réalisation de projets de territoire, la tendance à la concession (Giraut, 2006, p. 13) s'affirme au travers de l'identification de sites stratégiques pour l'accueil d'investissement étrangers. A titre d'exemples peuvent être citées les zones franches d'exportation Tanger Med au Maroc et de Bizerte en Tunisie. Ces espaces extraterritoriaux, cédés par l'Etat, échappent non seulement aux législations nationales en vigueur, mais aussi au contrôle par les collectivités territoriales et ainsi à toute initiative de développement territorial.

\section{Conclusion : limites et perspectives du développement territorial pour les Suds}

Les démarches de transfert de la thématique du développement territorial posent nombre de questions et, en premier lieu, celle du sens de la notion de « territoire » dans les pays du Maghreb. Quelle est l'utilité de ce concept? Quelles sont les conditions d'acceptation ou de rejet de cette catégorie d'analyse et de stratégie de développement transférée au Sud?

Les premiers résultats obtenus montrent que les autorités locales ont tendance à ne considérer que le territoire " donné », issu des organisations administratives hérités du 
colonialisme, au détriment des territoires " construits ", au travers de la coordination d'acteurs. Aussi, la notion de ressource territoriale devrait-elle être approfondie pour ces pays. Si les premières études ont été engagées au Maroc (Courlet, 2006), d'autres situations méritent d'être analysées. Cela pose l'importance des travaux de recherche comparatifs sur ces questions, impliquant les universités et équipes de recherche de chacun des pays.

Ces interrogations épistémologiques sous-tendent un questionnement sur les pratiques politiques et aménagistes: quelles peuvent être les définitions de "politiques de développement territorial » dans des pays où le sens même de territoire n'est souvent pas partagé, et où l'autonomie des acteurs territoriaux fait souvent défaut? La question est alors de savoir si le processus de transfert du modèle de " projet de territoire » peut favoriser l'autonomie nécessaire aux processus de construction et de mobilisation des ressources territoriales spécifiques.

La perspective essentielle réside dans un changement de culture, en passant des systèmes hiérarchisés à des systèmes publics, publics-privés, ou mixtes, hybrides ou coopératifs, traduisant une nouvelle capacité organisationnelle. Il s'agit en particulier d'accompagner la mise en place d' "opérateurs territorialisés » susceptibles de réaliser différents types d'opérations sur la ressource: révélation, protection, spécification, articulation avec d'autres ressources.

Dans le champ des modes de transfert, un certain nombre de conditions minimales peuvent être proposées pour mieux accompagner le développement territorial. En tout premier lieu, l'échelle du transfert doit être interrogée. Les transferts d'Etat à Etat doivent évoluer vers des coopérations décentralisées portant sur le champ du développement territorial, à partir d'expériences concrètes développées par des collectivités territoriales. Pour ce faire, la définition d'un cadre d'intervention intégrant des appuis financiers et organisationnels serait nécessaire. Il pourrait prévoir en particulier des modalités d'implication des Universités et des équipes de recherche locales dans les transferts, en favorisant le développement des formations. Cette dynamique contribuerait à la mise en réseau des acteurs du développement territorial, dans des dispositifs de recherche action définis au niveau local, national et international.

\section{BIBLIOGRAPHIE}

Abdelmalki L., Courlet C., 1996. Les nouvelles logiques du développemenT. Paris, L'harmattan, Logiques économiques. $415 \mathrm{p}$.

Angeon V., Houédété T., 2006. Le développement entre gouvernement et gouvernance : réflexion critique sur le rôle des institutions dans le développement. In Ferguène A. (éd.), Gouvernance locale et développement territorial. Le cas des pays du Sud. Paris, L'harmattan. 407 p.

Bouchrara M., 1987. L'industrialisation rampante, ampleur, mécanismes et portée. Exemples tunisiens. Economie et humanisme, $\mathrm{n}^{\circ} 296$. 
Bellaoui A., 2009. L'organisation régionales au Maroc : vers une plus grande artificialisation de l'espace géographique. In Boujrouf S., Antheaume B, Giraut F., Landel P.A., 2009, Les territoires à l'épreuve des normes : et référents et innovations. Contributions croisées sud africaines, françaises et marocaines. Coédition du Laboratoire des Etudes et de Recherches sur les Montagnes Atlasiques (LERMA), de la faculté des lettes et des sciences humaines, Université Cadi Ayyad, Marrakech, et de la revue Montagnes Méditerranéennes, 526 p. (p.79-92).

Colletis G., Courlet C., Pecqueur B., Winterhalter F., 1990. Les Systèmes industriels localisés. Grenoble, IREPD.

Colletis G., Pecqueur B., 1993. Intégration des espaces et quasi intégration des firmes : vers de nouvelles rencontres productives? Revue d'Economie Régionale et Urbaine, nº 3, p. 489-508.

Courlet C. (dir.), 2006. Territoire et développement économique au Maroc. Le cas des systèmes productifs localisés. Paris, Economie critique, L'harmattan.

Courlet C., 2008. L'économie territoriale. PUG, 135 p.

Courlet C., 1989. Les industrialisations endogènes. Revue Tiers-Monde, $\mathrm{n}^{\circ}$ 118, p. 413-421.

Courlet C., Gaulé A., 1974. Etude sur les surcoûts de l'industrie. Le cas de la metalmécanique. Note interne.

Courlet C., Judet, P., 1986. Industrialisation et développement : la crise des paradigmes. Revue Tiers Monde, 27 (107), p. 519-536.

Destanne de Bernis G., 1971. Les industries industrialisantes et les options algériennes. TiersMonde, tome12, $\mathrm{n}^{\circ}$ 47, Le tiers monde en l'an 2000, p. 545-563.

Dresch J., 1963. Industrialisation au Mahgreb. Maspéro, 269 p.

Ferguene A., Hsaini A., 1998. Développement endogène et articulation entre globalisation et territorialisation. Revue Région et Développement, 7, p. 1-38.

Friedmann, J., Weaver, C., 1979. Territory and Function. The Evolution of Regional Planning. London.

Giraut F., 2006. Etude comparée des politiques d'Aménagement du territoire et de développement régional dans les pays du Sud. Ministère des Affaires étrangères, DGCID, 133 p.

Garofoli G., 1986. Le développement périphérique en Italie. Economie et Humanisme, n² 289.

Gorgeu Y., Jenkins C., 1997. La charte de territoire, une démarche pour un projet de développement durable. Paris, La Documentation française, $311 \mathrm{p}$.

Judet P., 1989. Quand la Ruhr entre au Musée. Tiers Monde, 30 (118), p. 407-411.

Judet P., 1986. Les nouveaux pays industriels. Paris, Les éditions Ouvrières, Economie et humanisme, ed. revue et corrigée.

Gillard L., 1970. Le secteur comme concept théorique, essai de méso-analyse. Université Paris 1.

Gilly J-P., Leroux I., Wallet F., 2004. Gouvernance et proximité. In Zimmermann J.B., Pecqueur B., Economie de proximités, Paris, Hermès-Lavoisier, p. 187-206.

Keating M., Loughlin J., 1997. The Political Economy of Regionalism. Robert Schuman Centre.

Koop K. 2007. Les nouvelles fractures du monde et le défi pour la recherche sur les inégalités de développement. Les Cahiers du GEMDEV, nº 31, p. 182-195.

Landel P.A., 2007. Invention de patrimoines et créations de territoires. In Gumuchian, Pecqueur, La Ressource territoriale, Paris, Economica, Anthropos. 
Mahdi M., 2009. La tribu au secours du développement pastoral. In La tribu à l'heure de la globalisation, Etudes Rurales, 2009/2 (n¹84), 304 p.

Pecqueur B., 2005. Le développement territorial, une nouvelle approche des processus $\mathrm{du}$ développement pour les économies du Sud. In Antheaume B., Giraut F., Le territoire est mort, vive les territoires! Une (re)fabrication au nom du développement, Paris, IRD Edition, 384 p, p. 295-316.

Pecqueur B., 1989. Le développement local. Syros, 148 p.

Perroux F., 1950. Economic Space: Theory and Applications. Quarterly Journal of Economics, 64, p. 89-104.

Rauch, T., 1996. Ländliche Regionalentwicklung im Spannungsfeld zwischen Weltmarkt, Staatsmacht und kleinbäuerlichen Strategien. Verlag für Entwicklungspolitik, Saarbrűcken.

Rist G., 2007, [1996]. Le développement : histoire d'une croyance occidentale. Paris, Presses de Sc. Poles presses, $483 \mathrm{p}$.

Scott A.J., 2003. La poussée régionale : vers une géographie de la croissance dans les pays en développement. Géographie, Économie, Société, 5, p. 31-57.

Scott, A., Storper, M., 1991. Le développement régional reconsidéré. Espaces et Sociétés, n 66/67, p. 7-37.

Stöhr W.B., Taylor D.R.F., 1981. Development from Above or Below ? The Dialectics of Regional Planning in Developing Countries. Chicester et al.

Stöhr W.B, Töddling, F., 1977. Spatial Equity - Some Anti-Theses to Current Regional Development Doctrine. Papers in Regional Science, 38 (1), p. 33-53.

\section{NOTES}

1. 1956 en Tunisie et au Maroc, 1962 en Algérie.

2. Voire la notion de "region» dans le monde scientifique anglo-saxon, "territory" étant généralement utilisé pour désigner une entité spatiale dans sa dimension juridico-politique.

3. La DATAR a été créée en 1963. D’abord dénommée « Délégation à l'aménagement du territoire et à l'action régionale ", elle est devenue « Délégation interministérielle à l'aménagement et à la compétitivité des territoires " (DIACT), en 2005. Depuis 2009, elle est redevenue DATAR, avec un nouvel intitulé : «Délégation interministérielle à l'aménagement du territoire et à l'attractivité régionale »

4. En Tunisie, la planification indicative est orientée vers le développement parallèle de l'agriculture et de l'industrie, et, sous l'influence du syndicaliste Ben Salah, le plan « Perspectives décennales (1962-1971) " prévoit un développement résolument autocentré. Quant à la Libye, elle se repose rapidement sur une économie de rente pétrolière. En Algérie, De Bernis, en tant que conseiller du gouvernement algérien, promulgue "l'industrie industrialisante », stipulant que le développement passe par le soutien de l'industrie en amont des branches de croissance (Destanne de Bernis, 1971).

5. Une des particularités des politiques maghrébines de développement et d'aménagement, et notamment celles de l'Algérie et du Maroc, fut la forte influence de l'économiste français François Perroux, pionnier du développement à renommée internationale pour son modèle du développement à partir de pôles de croissance sectoriels et du développement centrifuge (1950) et fondateur de l'Institut des sciences économiques et mathématiques appliquées à Dakar.

6. Cette analyse est issue d'un programme de recherche intitulé "les processus de territorialisation en Méditerranée : processus d'émergence des territoires ruraux dans les pays 
méditerranéens qui a fait l'objet d'une coopération entre l'UMR PACTE de Grenoble et l'Institut Agronomique de Montpellier, impliquant des chercheurs de 10 pays du bassin méditerranéen, dans le cadre du réseau RAFAC (Réseau Agriculture Familiale Comparée en méditerranée), sous la direction de Pierre Campagne et Bernard Pecqueur. Pour les pays du Maghreb, 3 équipes sont intervenues: Pour l'Algérie, celle de Slimane Bedrani, Directeur du CREAD, pour le Maroc, celle de Mohamed Mahdi, de l'école Supérieure d'Agronomie de Mekhnès, et pour la Tunisie, celle de Mohammed Elloumi, de l'Institut National d'Agronomie de Tunis.

7. En effet, le partenariat impliquait les Ministères maghrébins de l'Aménagement du Territoire, au travers d'une Direction chargée d'assurer le pilotage des opérations. Les publics concernés sont prioritairement des cadres de l'Etat. De l'échelle centrale aux échelons déconcentrés, ils constituent la cible essentielle des programmes. Les transferts impliquent des experts français, fonctionnaires d'administrations centrales, enseignants chercheurs et cadres des collectivités territoriales. Des séminaires réguliers ont été organisés autour de quatre thématiques: le diagnostic de territoire, la prospective, la contractualisation et l'évaluation.

\section{RÉSUMÉS}

Depuis la décolonisation, plusieurs modèles de développement ont fait l'objet de transferts de la France vers les pays du Maghreb. La crise du développement industriel a ouvert la voie au développement local à partir des années 1980, et la puissance du mouvement de mondialisation a vu émerger le développement territorial. Depuis le début des années 2000, différents programmes de coopération ont été développés dans le domaine de l'aménagement sur cette thématique entre la France et le Maroc, puis la Tunisie, enfin l'Algérie.

Le concept de territoire apparaît central dans les politiques françaises de développement, sa pertinence mérite d'être discutée dans les pays du Maghreb. Il pose en particulier la question de la décentralisation des politiques publiques, et de la capacité des acteurs locaux à participer à la mise en place de systèmes de gouvernance territoriale, deux conditions indispensables à la construction de ressources territoriales spécifiques.

Since decolonisation, a succession of development models has been transferred from France towards North African countries. The failure of the industrial development approach on national level opened the way for local development in the late 1980's, and the power of globalisation dynamics recently gave birth to the "territorial development" model. This new approach towards (economic) development and local governance is actually transferred into regional planning practices of Morocco, Tunisia and Algeria on behalf of several French cooperation programs.

We here question the relevance of the application of the concept of territory in these North African countries - a concept which turned out to be a central one in French regional planning politics. We particularly analyse the two preconditions for the revealing of specific territorial resources: the decentralisation processes of North African public policies and the capacity of local actors to participate at territorial governance systems. 
INDEX

Mots-clés : décentralisation, développement local, développement territorial, modèle de développement, ressource territoriale, transfert

Keywords : decentralisation, development model, local development, territorial development, territorial resources, transfer

\section{AUTEURS}

\section{KIRSTEN KOOP}

Kirsten Koop est Maître de conférences à l'université Joseph Fourier - Grenoble 1 et membre de l'UMR PACTE - Territoires. kirsten.koop@ujf-grenoble.fr

\section{PIERRE-ANTOINE LANDEL}

Pierre-Antoine Landel est Maître de conférences à l'université Joseph Fourier - Grenoble 1 et membre de l'UMR PACTE - Territoires. pierre-antoine.landel@ujf-grenoble.fr

\section{BERNARD PECQUEUR}

Bernard Pacqueur est Professeur à l'université Joseph Fourier - Grenoble 1 et membre de l'UMR PACTE - Territoires. Bernard.Pecqueur@ujf-grenoble.fr 